\title{
Black-Blood Imaging of the Human Heart Using Rapid Stimulated Echo Acquisition Mode (STEAM) MRI
}

\author{
Alexander Karaus, Dipl Phys, ${ }^{1}$ Klaus-Dietmar Merboldt, PhD, ${ }^{1}$ \\ Joachim Graessner, MSEE, ${ }^{2}$ and Jens Frahm, $\mathrm{PhD}^{1 *}$
}

\begin{abstract}
Purpose: To develop a rapid stimulated echo acquisition mode (STEAM) MRI technique for "black-blood" imaging of the human heart that overcomes the single-slice limitation and partially compromised blood suppression associated with double inversion-recovery techniques.
\end{abstract}

Materials and Methods: Black-blood multislice images of the heart along anatomic orientations and triggered to end diastole were obtained from healthy human subjects at 3T using rapid STEAM MRI sequences with five-eighths partial Fourier encoding and variable flip angles. Single-shot STEAM images at $2.5 \times 2.5 \mathrm{~mm}^{2}$ in-plane resolution and 6-mm section thickness were recorded in $230 \mathrm{msec}$ from individual heartbeats. Improved signal-to-noise ratio (SNR) and higher spatial resolution of $2.0 \times 2.0 \mathrm{~mm}^{2}$ and $1.5 \times$ $1.5 \mathrm{~mm}^{2}$ were achieved by segmented multishot STEAM MRI with interleaved $\mathrm{k}$-space acquisitions (160 msec each) from several heartbeats. In a single breathhold covering 18 heartbeats selected applications employed either three segments with six sections or six segments with three sections.

Results: Because stimulated echoes (STEs) dephase signals from moving spins, rapid STEAM images are free from blood signal contamination. The method offers a flexible tradeoff between spatial resolution, imaging speed (i.e., number of segments), and volume coverage (i.e., number of sections).

Conclusion: Rapid STEAM MRI of the heart emerges as a simple technique for multislice imaging of the myocardial wall with efficient flow suppression.

Key Words: heart; magnetic resonance imaging; cardiac imaging; rapid imaging; STEAM; black-blood imaging; 3T

J. Magn. Reson. Imaging 2007;26:1666-1671.

(c) 2007 Wiley-Liss, Inc.

ALTHOUGH TREMENDOUS ADVANCES have been made in cardiac MRI with respect to dynamic imaging of myocardial blood flow and wall motion, specific appli-

${ }^{1}$ Biomedizinische NMR Forschungs GmbH am Max-Planck-Institut fü biophysikalische Chemie, Göttingen, Germany.

${ }^{2}$ Siemens AG Medical Solutions, Hamburg, Germany.

*Address reprint requests to: J.F., Biomedizinische NMR Forschungs GmbH, 37070 Göttingen, Germany. E-mail: jfrahm@gwdg.de

Received April 6, 2007; Accepted September 6, 2007.

DOI 10.1002/jmri.21208

Published online 26 October 2007 in Wiley InterScience (www. interscience.wiley.com). cations still deserve methodologic attention, e.g., see articles in a special issue of JMRI (1) and a more recent report on progress and challenges (2). This particularly holds true for "black-blood" imaging of the myocardial wall, which aims at an assessment of its structural (or functional) integrity without interference from MRI signals of the blood. Conventional methods for depiction of cardiac morphology such as the double inversion-recovery technique $(3,4)$ attempt to suppress the blood by nonselective preinversion of the MRI signal of the entire heart followed by slice-selective reinversion of the target section. Subsequently, data acquisition of the center of $\mathrm{k}$-space is performed at a time when the signal from the inflowing blood is more or less nulled. While the image quality achieved in practice largely depends on the inversion time, imaging time, and stability of the heart rate, the approach may also lower the signal-to-noise ratio (SNR) of the desired signal from the myocardial wall and, most importantly, limits the number of simultaneously-acquired sections in a multislice study, e.g., see Refs. 5 and 6 for recent improvements aiming at flow-suppressed vessel wall imaging.

The present work focuses on an alternative technique based on the acquisition of a single-shot stimulated echo acquisition mode (STEAM) image (7). Although the possible usefulness of flow-suppressed STEAM MRI of the heart has already been recognized as early as 1991 (8), only more recent advances in MRI hardware allowed for a renewed interest in practical applications, for example see Refs. 9 and 10. Specific technical improvements refer to the strength of the magnetic field, gradient performance, and availability of multiple receiver coils and channels. The purpose of this study was to redesign a rapid STEAM MRI technique with use of state-of-the-art equipment to evaluate its potential for blood-free multislice imaging of the myocardial wall.

\section{MATERIALS AND METHODS}

\section{Rapid STEAM MRI}

The basic STEAM MRI sequence employs three sliceselective radio frequency $(\mathrm{RF})$ pulses with preferentially 
$90^{\circ}$ flip angles (11). An initial $90^{\circ} \mathrm{RF}$ pulse excites transverse magnetization, which after an interval TE/2 becomes retransformed into longitudinal magnetization by a second $90^{\circ} \mathrm{RF}$ pulse. During the subsequent middle interval (TM) this magnetization is subject to $\mathrm{T} 1$ relaxation before it is read out by a third $\mathrm{RF}$ pulse generating a STE after another period TE/2. During TE, the transverse magnetization leading to the STE is subject to $\mathrm{T} 2$ relaxation.

A high-speed version of the STEAM sequence may be obtained by distributing the longitudinal magnetization prepared by the leading two RF pulses into multiple portions. This may be accomplished by replacing the third $90^{\circ} \mathrm{RF}$ pulse by a rapid series of readout $\mathrm{RF}$ pulses with flip angles lower than $90^{\circ}$ (7). The corresponding series of stimulated echoes (STEs) may then be differently phase-encoded similar to a fast spin-echo or rapid acquisition with relaxation enhancement (RARE) MRI sequence. A schematic diagram of a singleshot STEAM MRI sequence optimized for cardiac applications is shown in Fig. 1. Selection and definition of the target section is mainly accomplished by the first sinc-shaped $90^{\circ} \mathrm{RF}$ pulse, which in the current implementation had a duration of $2.56 \mathrm{msec}$ and an optimum number of side lobes (i.e., a bandwidth-time product of 5.4). To maintain the quality of the achieved slice profile as much as possible, that is by maximum refocusing across the slice dimension, the second $90^{\circ} \mathrm{RF}$ pulse and the low-flip angle readout pulses were shortened to a duration of $0.64 \mathrm{msec}$ (sinc-shaped, bandwidth-time product of 1.7).

The frequency-encoding read gradient in each TR interval is predephased in the very first TE/ 2 interval and serves to fulfill the STE condition, that is full dephasing of the excited transverse magnetization prior to the application of the second RF pulse (11). Unwanted echo formation was most effectively prevented by a strong spoiler gradient $\left(20 \mathrm{mT} \mathrm{m}^{-1}\right)$ along the read gradient axis during the first middle interval TM (duration 5 msec). To preclude the establishment of unwanted transverse coherences during the repetitive readout in-

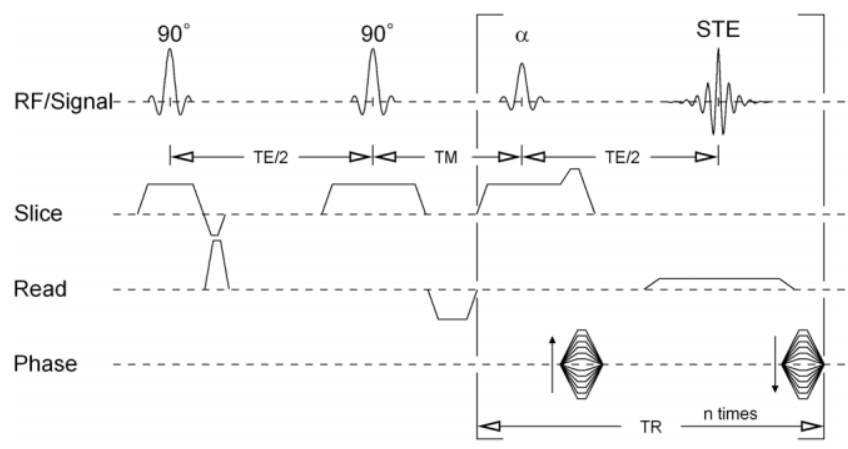

Figure 1. Schematic RF pulse and gradient diagram for rapid STEAM MRI of the heart. The slice-selective longitudinal magnetization prepared by the first two $90^{\circ} \mathrm{RF}$ pulses is read out as a series of STEs using low-flip angle RF pulses $(\alpha)$; TE $=$ echo time of the STE, TM = first middle interval, TR $=$ duration of the repetitive readout interval, $N=$ number of phase-encoding steps (or readout intervals). For further details see text and Table 1. tervals TR, every phase-encoding step was rephased immediately after data acquisition and the phase of the low-flip angle readout pulses was subjected to RF spoiling.

Moreover, the sequence employed a linear ordering of the phase-encoding steps, five-eighths partial Fourier encoding, and variable flip angles to maximize the SNR without affecting the point-spread function along the phase-encoding dimension of the image. This may be achieved by generating STEs with similar intensity and requires an iterative calculation of successive flip angles such that the last flip angle reaches $90^{\circ}$ (7). Image reconstruction was accomplished with use of a projection onto convex subsets (POCS) algorithm as previously described (12). Additional options not visualized in Fig. 1 include electrocardiograph (ECG) synchronization, frequency-selective fat suppression by a preceding chemical shift selective (CHESS) pulse and spoiler gradient (13), and spatial presaturation modules.

\section{Subjects}

A total of six male volunteers (age range 20-32 years) with no known disease participated in the study; several subjects were examined multiple times. Written informed consent was obtained in all cases prior to the examination. All experimental procedures conformed fully to institutional guidelines.

\section{MRI Experiments}

Rapid STEAM MRI sequences were implemented on a 3T MRI system (Magnetom Tim Trio; Siemens, Erlangen, Germany) equipped with a 32-channel receiver system and $40 \mathrm{mT} \mathrm{m}^{-1}$ gradients. Heart images were recorded with use of a six-element body matrix coil in combination with six or nine elements of a 24-element spine matrix coil. Data acquisition was ECG-synchronized to end diastole and multislice studies were performed during a single breathhold in expiration. Fat suppression was applied in all cases. Mild postprocessing involved adaptive filtering (predefined medium edge enhancement and smoothing, software supplied by the manufacturer), which takes into account the local intensity distribution and the continuation of local tissue structures.

Typically, image sections had a thickness of $6 \mathrm{~mm}$ and covered a rectangular field of view (FOV) of about $200 \times 320 \mathrm{~mm}^{2}$. Single-shot STEAM images at $2.5 \times$ $2.5 \mathrm{~mm}^{2}$ in-plane resolution were acquired with a receiver bandwidth of $500 \mathrm{~Hz} /$ pixel and a matrix resolution of $80 \times 128$, which was reduced by five-eighths partial Fourier encoding to $50 \times 128$. Flip angles of the corresponding 50 STEs increased from $7.9^{\circ}$ (first echo) to $90^{\circ}$ (last echo). The resulting STE time was $\mathrm{TE}=4.56$ msec and the duration of the readout interval was TR $=$ $4.16 \mathrm{msec}$. Together with the time needed for fat suppression (about $6 \mathrm{msec}$ ), the leading two RF pulses, the first $\mathrm{TE} / 2$ interval, and the middle interval $\mathrm{TM}=5$ msec, the total measurement time yielded $233 \mathrm{msec}$.

A higher in-plane resolution was obtained with use of a segmented multishot STEAM MRI technique that acquired interleaved $\mathrm{k}$-space portions (rather than contig- 
Table 1

Imaging Parameters for Rapid STEAM MRI of the Human Heart

\begin{tabular}{|c|c|c|c|c|}
\hline & \multicolumn{4}{|c|}{ Number of segments } \\
\hline & \multirow{2}{*}{$\begin{array}{c}\text { Single-shot } \\
\text { STEAM } \\
\text { MRI } \\
1\end{array}$} & \multicolumn{3}{|c|}{ Multishot STEAM MRI } \\
\hline & & 3 & 6 & 9 \\
\hline Resolution $\left(\mathrm{mm}^{2}\right)$ & $2.5 \times 2.5$ & $2.0 \times 2.0$ & $1.5 \times 1.5$ & $1.0 \times 1.0$ \\
\hline $\mathrm{FOV}\left(\mathrm{mm}^{2}\right)$ & $200 \times 320$ & $208 \times 320$ & $216 \times 312$ & $224 \times 320$ \\
\hline Base resolution & $80 \times 128$ & $104 \times 160$ & $144 \times 208$ & $224 \times 320$ \\
\hline Acquisition matrix ${ }^{a}$ & $50 \times 128$ & $65 \times 160$ & $90 \times 208$ & $140 \times 320$ \\
\hline Number of Lines/segment & 50 & 22 & 15 & 16 \\
\hline First flip angle $\left(^{\circ}\right)$ & 7.9 & 12.2 & 14.9 & 14.4 \\
\hline Bandwidth ( $\mathrm{Hz}$ pixel $\left.{ }^{-1}\right)$ & 500 & 250 & 150 & 150 \\
\hline Echo time (msec) & 4.56 & 6.52 & 9.18 & 9.18 \\
\hline Readout interval (msec) & 4.16 & 6.19 & 8.92 & 9.07 \\
\hline Imaging time ${ }^{\mathrm{b}}$ (msec) & 233 & 162 & 161 & 172 \\
\hline
\end{tabular}

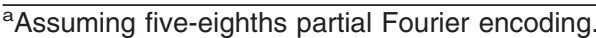

bIncluding fat suppression.

uous blocks) from several heartbeats. In comparison to single-shot variants and despite the use of a larger acquisition matrix, the approach allowed for a reduction of the number of simultaneously acquired phaseencoding steps per heartbeat (i.e., Fourier lines per segment), which in turn increased the flip angles of the readout RF pulses and improved the overall SNR. Moreover, the concomitant reduction of the acquisition time for an individual segment led to a more robust behavior with respect to variable heart rates.

For a single breathhold covering 18 heartbeats, selected applications employed either three segments with six sections at $2.0 \times 2.0 \mathrm{~mm}^{2}$ resolution or six

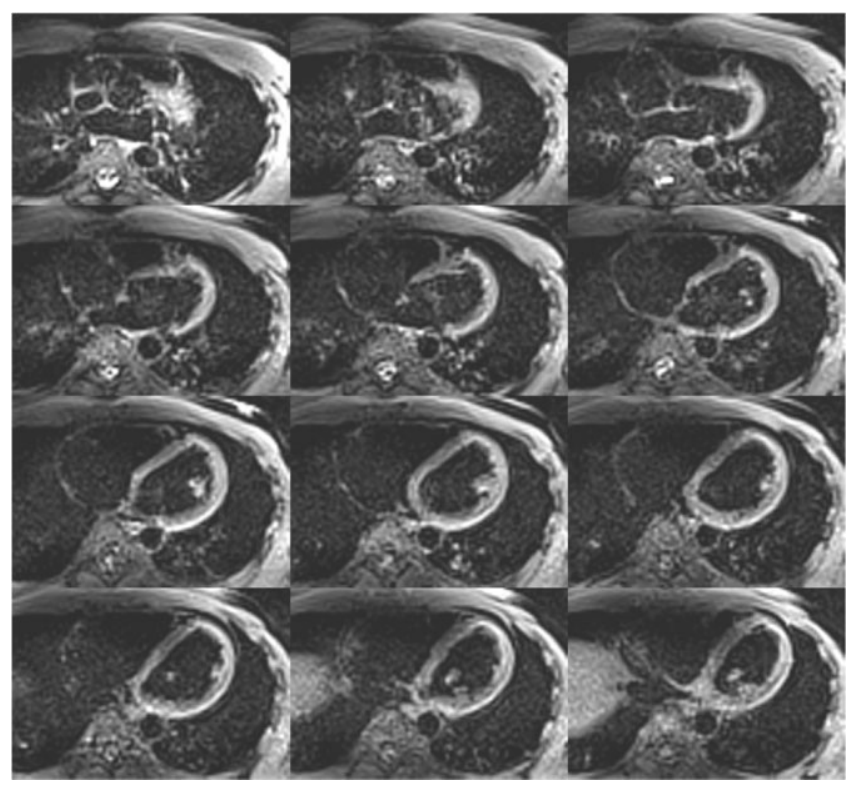

Figure 2. Contiguous single-shot STEAM images of the human heart (transverse views from top to bottom) at 2.5-mm in-plane resolution. A total of 12 fat-suppressed sections at 6-mm thickness were acquired from 12 heartbeats in a single breathhold in expiration (233 msec each). Data acquisition was ECG-synchronized to end-diastole (delay to $\mathrm{R}$ wave $=700$ msec, mean heart rate $=50$ ). For other parameters see Table 1 . segments with three sections at $1.5 \times 1.5 \mathrm{~mm}^{2}$ resolution. Typical imaging parameters are listed in Table 1 together with those used for a single-shot STEAM MRI sequence and a variant based on nine segments for cardiac imaging at $1.0 \times 1.0 \mathrm{~mm}^{2}$ in-plane resolution.

\section{RESULTS}

Figure 2 shows 12 transverse single-shot STEAM images of the heart of a human volunteer acquired in a
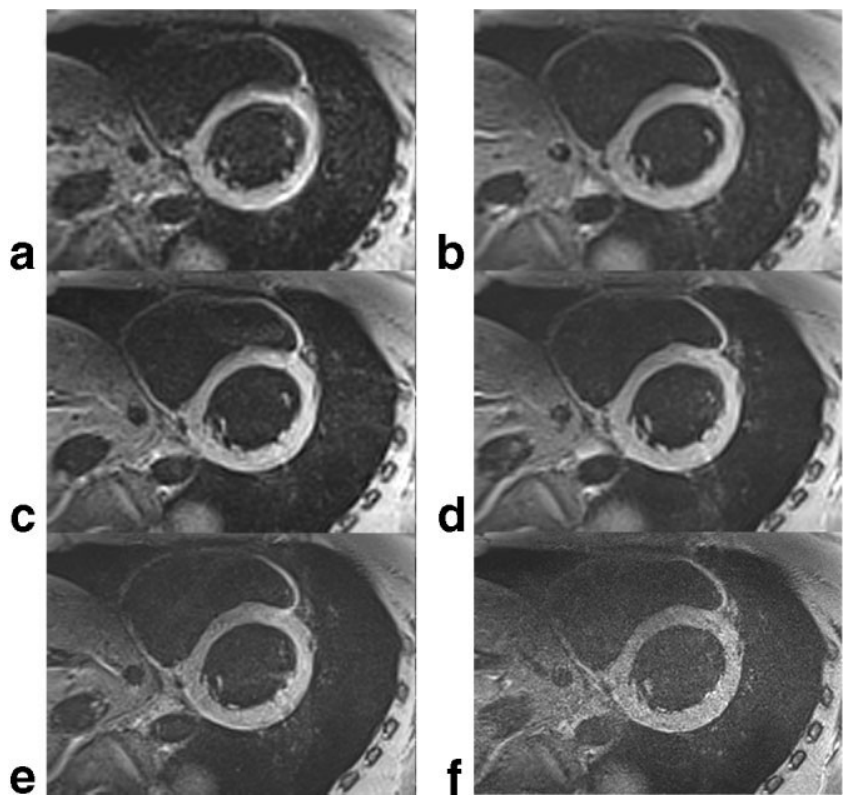

Figure 3. Rapid STEAM images of the human heart (shortaxis views with fat suppression) at different in-plane resolutions. a: Single-shot image at $2.5-\mathrm{mm}$ resolution $(233 \mathrm{msec})$. b: Segmented three-shot image at $2.5-\mathrm{mm}$ resolution $(3 \times 96$ msec). c: Segmented three-shot image at $2.0 \mathrm{~mm}$ resolution $(3 \times 162 \mathrm{msec})$. d: Segmented six-shot image at $2.0-\mathrm{mm}$ resolution $(6 \times 125 \mathrm{msec})$. e: Segmented six-shot image at $1.5-\mathrm{mm}$ resolution $(6 \times 161 \mathrm{msec})$. f: Segmented nine-shot image at $1.0-\mathrm{mm}$ resolution $(9 \times 172 \mathrm{msec})$. For other parameters see Table 1. 
Figure 4. Contiguous rapid STEAM images of the human heart at $2.0-\mathrm{mm}$ resolution (short-axis views from base to apex). A total of 12 fat-suppressed sections of $6-\mathrm{mm}$ thickness were obtained from two breathholds with use of a segmented three-shot STEAM MRI sequence yielding six sections in 18 heartbeats. For other parameters see Table 1 .

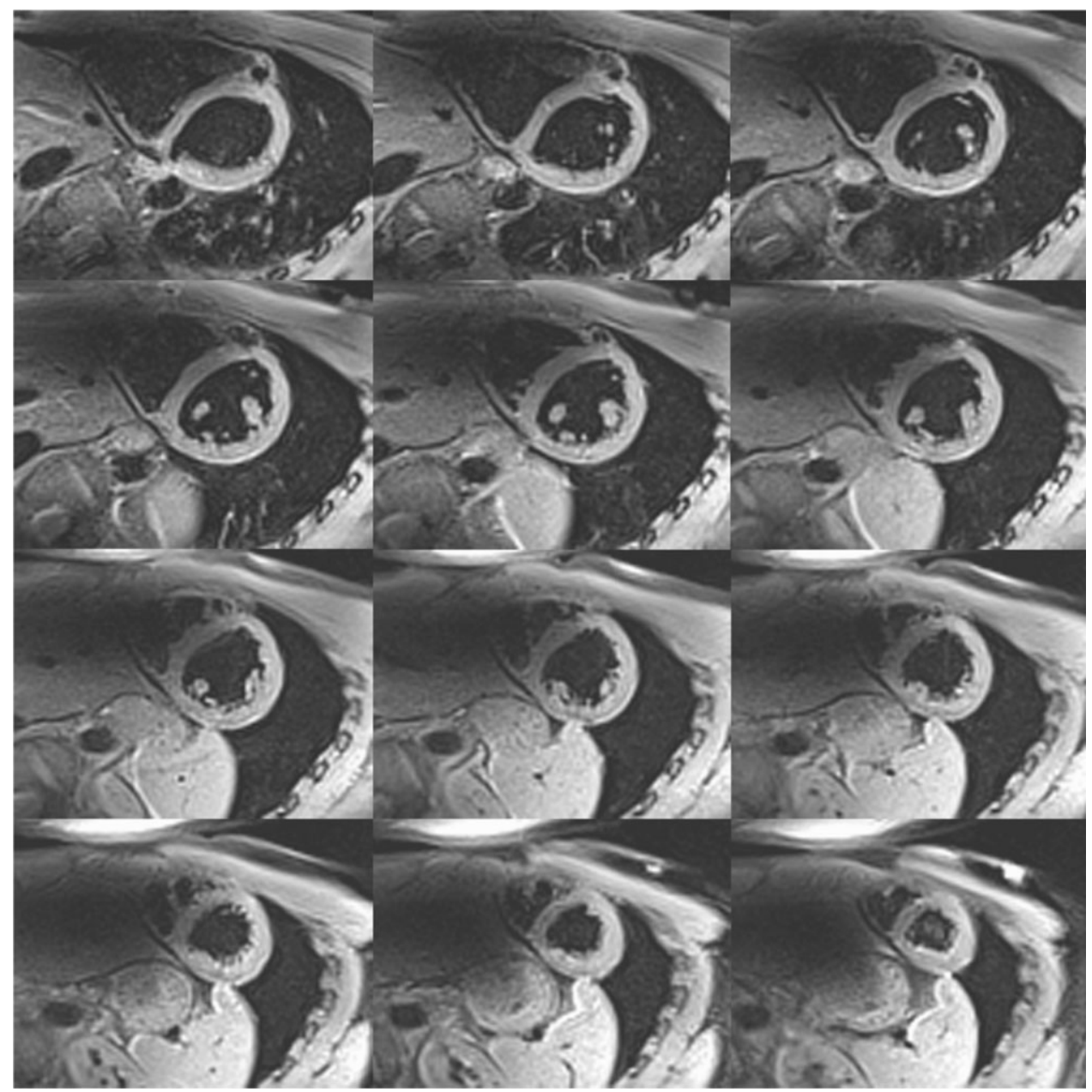

single breathhold (12 heartbeats). Each fat-suppressed section at 2.5-mm in-plane resolution and 6-mm thickness was acquired in $233 \mathrm{msec}$ from a late diastolic phase. As most volunteers presented with heart rates in the range of 50-55, the typical delay to the $\mathrm{R}$ wave was 700 msec. While these images certainly demonstrate the effective suppression (dephasing) of blood signals, they also reveal a limited SNR and resolution. Therefore, Fig. 3 presents short-axis views of a different subject, which compare a single-shot STEAM image (Fig. 3a) with images obtained by segmented multishot STEAM MRI (Fig. 3b-f). The improved SNR, higher resolution, and reduced acquisition time for individual segments are at the expense of prolonging the overall measurement to at least as many heartbeats as segments required. In fact, in order to efficiently exploit the time of a single breathhold (here kept to 18 heartbeats) all applications were set up as interleaved multislice acquisitions using the end-diastolic phase of each heartbeat.

In more detail, the results of Fig. 3 indicate the limited performance of a single-shot 2.5-mm image (Fig. 3a) as well as the borderline SNR of an acquisition at 1.0-mm resolution (Fig. 3f). On the other hand, a comparison of images with identical resolution but different degrees of segmentation clearly demonstrates a substantial gain in SNR for a larger number of segments. This holds true for 2.5-mm images in going from a single-shot (Fig. 3a) to a three-shot version (Fig. 3b) as well for 2.0-mm images when moving from a three-shot (Fig. 3c) to a six-shot version (Fig. 3d). For practical purposes, the most promising strategies are a segmented three-shot STEAM MRI sequence at $2.0-\mathrm{mm}$ resolution (Fig. 3c) and a segmented six-shot STEAM MRI sequence at $1.5-\mathrm{mm}$ resolution (Fig. 3e). Corresponding short-axis views (12 contiguous sections) from two breathholds $(2 \times 6$ sections at $2.0-\mathrm{mm}$ resolution) and four breathholds ( $4 \times 3$ sections at $1.5-\mathrm{mm}$ resolution) are summarized in Figs. 4 and 5, respectively. In addition, Fig. 6 depicts two-chamber and fourchamber views (three sections) at $2.0-\mathrm{mm}$ and $1.5-\mathrm{mm}$ in-plane resolution acquired in either nine or 18 heartbeats.

\section{DISCUSSION}

Rapid STEAM images of the human heart are free from any contamination by flowing blood. In fact, it should be noted that a clear delineation of the heart muscle required ECG synchronization to end diastole as movements during data acquisition may corrupt the resulting image. The problem has previously been noticed and effectively been treated by Fahmy et al (10) using a dual-gradient modulation for cine applications. In this study, a poor definition of sharp features such as a thin myocardial wall was eventually noticed for single-shot 


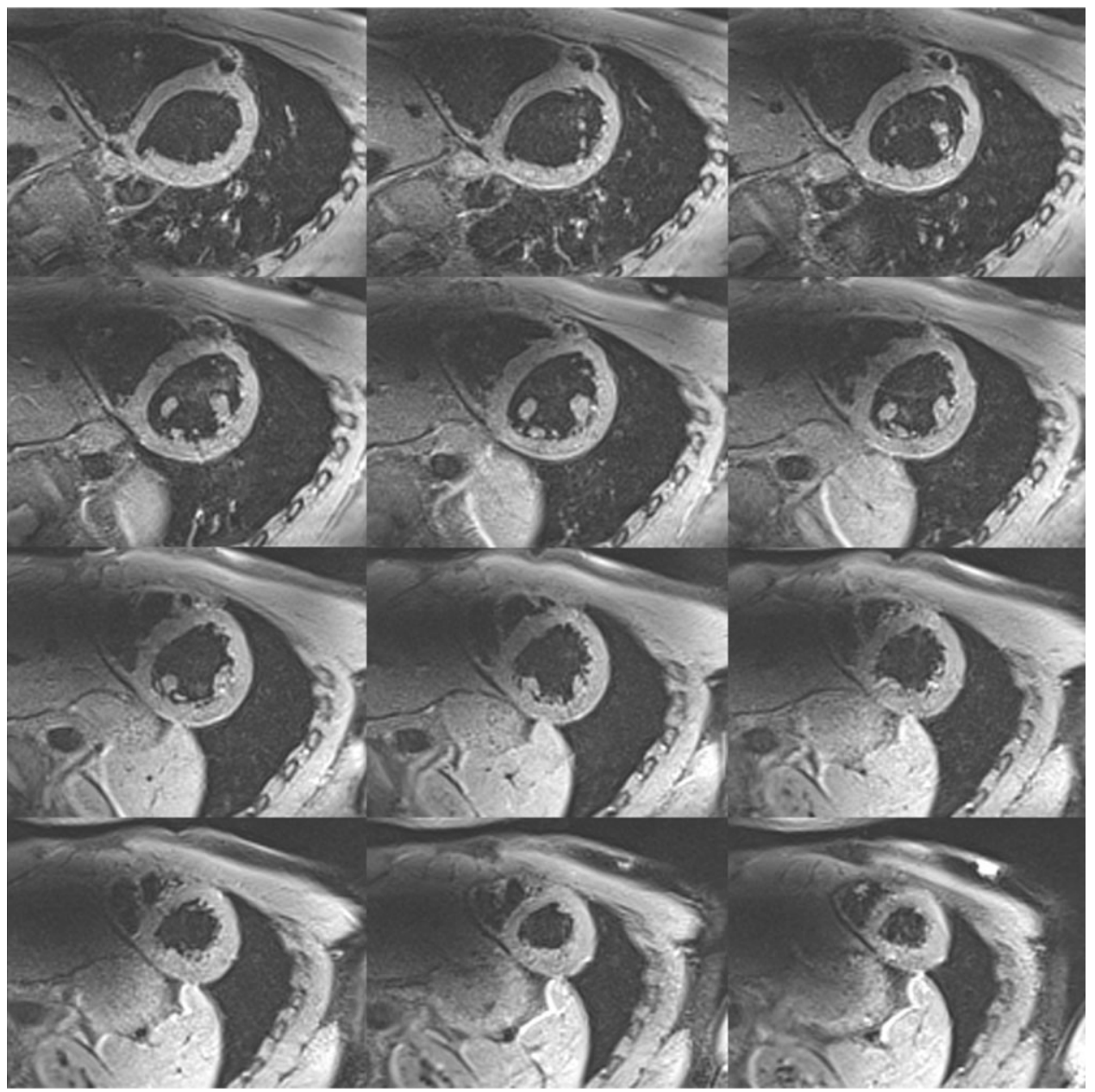

Figure 5. Contiguous rapid STEAM images of the human heart at $1.5-\mathrm{mm}$ resolution (short-axis views from base to apex, same subject as in Fig. 4). A total of 12 fat-suppressed sections of $6-\mathrm{mm}$ thickness were obtained from four breathholds with use of a segmented six-shot STEAM MRI sequence yielding three sections in 18 heartbeats. For other parameters see Table 1 .
STEAM MRI when the data acquisition extended into the next heartbeat because of an unexpectedly short RR interval. The physical reason is the loss of high spatial frequency information for image reconstruction.

Because the signal strength of successive STEs in a rapid STEAM sequence is attenuated by $\mathrm{T} 1$ relaxation, the soft-tissue contrast of the images is predominantly given by $\mathrm{T} 1$ (and spin density). Rather than being imposed by partial saturation in a repetitive MRI sequence, the $\mathrm{T} 1$ contrast in rapid STEAM MRI behaves in close analogy to the T2 contrast in fast spin-echo MRI. Its diagnostic usefulness for the detection of heart pathology remains to be evaluated, but it seems conceivable that the sequence offers considerable potential for the elucidation of T1-weighted signal intensity changes induced by the differential uptake of contrast media.

Based on these preliminary results, rapid STEAM MRI of the heart presents as a simple technique for unrestricted multislice imaging of the myocardial wall with efficient flow suppression. While these features are certainly advantageous in comparison to double inversion-recovery techniques, it should be emphasized that the present work only deals with the basic methodologic development and first proof-of-principle applications to healthy subjects. Prior to clinical applications the achieved image quality needs to be further established and more quantitative comparisons are warranted, for example with single-shot fast spin-echo (RARE) imag- ing of the heart $(14,15)$. In fact, STEs possess only half the intensity of a comparable spin echo, but rapid multislice RARE imaging may become critical with respect to RF power deposition at high magnetic fields. On the other hand, the prolonged $\mathrm{T} 1$ relaxation times of myocardial muscle at higher fields will be beneficial for rapid STEAM MRI, whereas the concomitantly decreased T2 relaxation times impose even stronger restrictions to the echo train length of a RARE sequence. Corresponding deficiencies in terms of poor contrast and image blurring have already been noticed (2).

In conclusion, rapid STEAM MRI sequences allow for black-blood imaging of the cardiac morphology in arbitrary orientations and with an in-plane resolution of up to $1.0 \mathrm{~mm}$. However, a particularly promising approach is provided by a segmented six-shot version yielding three multislice images at $1.5-\mathrm{mm}$ resolution within 18 heartbeats. The current implementation involves enddiastolic data acquisitions of about $160 \mathrm{msec}$ duration including fat suppression.

Alternative improvements in spatial resolution may be obtained by reducing the FOV in phase-encoding direction by the incorporation of a second slab-selective excitation orthogonal to the slice-selection direction. This functionality may be imposed onto the second $90^{\circ}$ $\mathrm{RF}$ pulse of the rapid STEAM MRI sequence without any penalty in acquisition time. It offers the direct recording of "zoomed" images without aliasing. At the same time, 


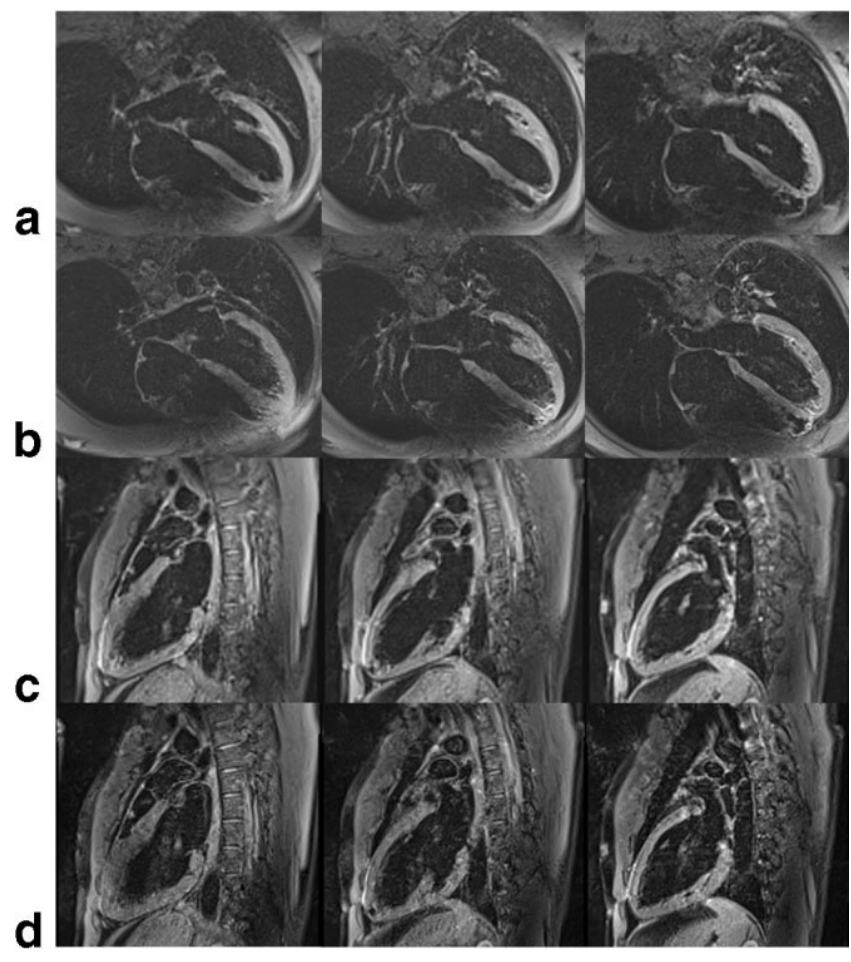

Figure 6. Rapid STEAM images of the human heart depicting multislice $(\mathbf{a}, \mathbf{b})$ four-chamber views and $(\mathbf{c}, \mathbf{d})$ two-chamber views. Three fat-suppressed sections of 6-mm thickness (3-mm gaps) were obtained from a single breathhold with use of $(\mathrm{a}, \mathrm{c})$ a segmented three-shot STEAM MRI sequence at 2.0-mm resolution (nine heartbeats) and (b,d) a segmented six-shot version at $1.5-\mathrm{mm}$ resolution (18 heartbeats). For other parameters see Table 1 .

the simultaneous reduction of the number of phaseencoding steps promises even shorter measuring times and improved SNR because of the possibility of higher flip angles for a reduced number of readout RF pulses. Similar gains in performance are to be expected by adopting the principles of parallel imaging for rapid STEAM MRI. Moreover, improved spatial resolution may also be obtained by the use of radial encoding for single-shot STEAM MRI (16), because acquisitions with pronounced undersampling may be successfully treated with advanced iterative image reconstruction principles (17).

\section{REFERENCES}

1. Higgins CB, editor. Special issue: cardiovascular MRI. J Magn Reson Imaging 1999;10:589-899.

2. Earls JP, Ho VB, Foo TK, Castillo E, Flamm SD. Cardiac MRI: recent progress and continued challenges. J Magn Reson Imaging 2002; 16:111-127.

3. Edelmann RR, Chien D, Kim D. Fast selective black blood MR imaging. Radiology 1991;181:655-660.

4. Simonetti OP, Finn JP, White RD, Laub G, Henry D. "Black blood" T2-weighted inversion-recovery MR imaging of the heart. Radiology 1996;199:49-57.

5. Yarnykh VL, Yuan C. Multislice double inversion-recovery blackblood imaging with simultaneous slice reinversion. J Magn Reson Imaging 2003; 17:478-483.

6. Itskovich VV, Mani V, Mizsei G, et al. Parallel and nonparallel simultaneous multislice black-blood double inversion recovery techniques for vessel wall imaging. J Magn Reson Imaging 2004; 19:459-467.

7. Frahm J, Haase A, Matthaei D, Merboldt K, Hänicke W. Rapid NMR imaging using stimulated echoes. J Magn Reson 1985;65:130-135.

8. Frahm J, Hänicke W, Bruhn H, Gyngell M, Merboldt K. High-speed STEAM MRI of the human heart. Magn Reson Med 1991;22:133142.

9. Aletras $\mathrm{AH}$, Arai AE. Meta-DENSE complex acquisition for reduced intravoxel dephasing. J Magn Reson 2004;169:246-249.

10. Fahmy AS, Pan L, Osman NF. Artifact-free black-blood cine cardiac imaging in a single breath-hold. Magn Reson Imaging 2006;24: 1303-1310.

11. Frahm J, Merboldt KD, Hänicke W, Haase A. Stimulated echo imaging. J Magn Reson 1985;64:81-93.

12. Rieseberg S, Merboldt KD, Küntzel M, Frahm J. Diffusion tensor imaging using partial Fourier STEAM MRI with projection onto convex subsets reconstruction. Magn Reson Med 2005;54:486-490.

13. Haase A, Frahm J, Hänicke W, Matthaei D. ${ }^{1} \mathrm{H}$ NMR chemical shift selective (CHESS) imaging. Phys Med Biol 1985;30:341-344.

14. Le Roux P, Gilles RJ, McKinnon CG, Carlier P. Optimized outer volume suppression for single-shot fast spin-echo cardiac imaging. J Magn Reson Imaging 1998;8:1022-1032.

15. Vignaux OB, Augui J, Coste J, et al. Comparison of single-shot fast spin-echo and conventional spin-echo sequences for MR imaging of the heart: initial experience. Radiology 2001;219: 545-550.

16. Block KT, Frahm J. Radial single-shot STEAM MRI. Magn Reson Med 2007; in press.

17. Block KT, Frahm J. Undersampled radial MRI with multiple coils. Iterative image reconstruction using a total variation constraint. Magn Reson Med 2007;57:1086-1098. 